\title{
Norm and Taylor coefficients estimates of holomorphic functions in balls
}

\author{
by Jacob Burbea (Pittsburgh, Penn.) and Do Young KwaK* (Taejon)
}

\begin{abstract}
A classical result of Hardy and Littlewood states that if $f(z)=$ $\sum_{m=0}^{\infty} a_{m} z^{m}$ is in $H^{p}, 0<p \leq 2$, of the unit disk of $\mathbb{C}$, then $\sum_{m=0}^{\infty}(m+1)^{p-2}\left|a_{m}\right|^{p} \leq$ $c_{p}\|f\|_{p}^{p}$ where $c_{p}$ is a positive constant depending only on $p$. In this paper, we provide an extension of this result to Hardy and weighted Bergman spaces in the unit ball of $\mathbb{C}^{n}$, and use this extension to study some related multiplier problems in $\mathbb{C}^{n}$.
\end{abstract}

1. Notation and preliminaries. For $z=\left(z_{1}, \ldots, z_{n}\right)$ and $\zeta=\left(\zeta_{1}, \ldots\right.$ $\left.\ldots, \zeta_{n}\right)$ in $\mathbb{C}^{n}$ and for $\alpha=\left(\alpha_{1}, \ldots, \alpha_{n}\right)$ in $\mathbb{Z}_{+}^{n}$, we use the standard notation of $\langle z, \zeta\rangle=z_{1} \bar{\zeta}_{1}+\ldots+z_{n} \bar{\zeta}_{n},\|z\|^{2}=\langle z, z\rangle, z^{\alpha}=z_{1}^{\alpha_{1}} \ldots z_{n}^{\alpha_{n}}, \alpha !=\alpha_{1} ! \ldots \alpha_{n}$ ! and $|\alpha|=\alpha_{1}+\ldots+\alpha_{n}$. Then $B=B_{n}=\left\{z \in \mathbb{C}^{n}:\|z\|<1\right\}$ is the unit ball of $\mathbb{C}^{n}$ and $\Delta=B_{1}$ is the unit disk of $\mathbb{C}$. The normalized surface measure on the boundary $\partial B$ of $B$ is denoted by $d \sigma$, and the volume euclidean measure is denoted by $d v$. We consider a family of probability measures $d v_{q}, q \geq 0$, on $\bar{B}$ defined by $d v_{0}=d \sigma$ and

$$
d v_{q}(z)=\frac{1}{\pi^{n}} \frac{\Gamma(n+q)}{\Gamma(q)}\left(1-\|z\|^{2}\right)^{q-1} d v(z)
$$

when $q>0$.

For $0<p \leq \infty$ and $q \geq 0$, the $L^{p}$-space with respect to $d v_{q}$ is denoted by $L_{q}^{p}=L_{q}^{p}(B)$, and the corresponding quasi-norm is denoted by $\|\cdot\|_{p, q}$. We also let $\langle,\rangle_{q}$ denote the inner product of the Hilbert space $L_{q}^{2}$. The class of all holomorphic functions on a domain $D$ in $\mathbb{C}^{n}$ is denoted by $H(D)$. For a function $f$ on $B$, and $0<r<1$, we let $f_{r}(z)=f(r z), z \in \bar{B}$, $M_{\infty}(f: r)=\sup \left\{\left|f_{r}(z)\right|: z \in \partial B\right\}$ and

$$
M_{p}(f: r)=\left\{\int\left|f_{r}\right|^{p} d v_{0}\right\}^{1 / p} \quad(0<p<\infty) .
$$

1991 Mathematics Subject Classification: 32A35, 32H10, 42B10, 42B15, 42B30, 46J15. *Partially supported by KOSEF. 
We let $A_{q}^{p}=A_{q}^{p}(B)$ denote the subspace of $L_{q}^{p}$ consisting of functions in $H(B)$. In particular, $A_{0}^{p}$ is the Hardy class $H^{p}$ which we identify in the usual way as a subspace of $L_{0}^{p}=L^{p}(\partial B)$. Moreover, for any $\mathrm{f}$ in $H(B)$ we have

$$
\|f\|_{p, 0}=\sup _{0<r<1} M_{p}(f: r)
$$

and the norms $\|f\|_{p, q}$ converge to $\|f\|_{p, 0}$ as $q \rightarrow 0^{+}$. This shows that $A_{0}^{p}=H^{p}$ may be viewed as a decreasing limit of the weighted Bergman spaces $A_{q}^{p}$ as $q \rightarrow 0^{+}$.

Evidently, $A_{q}^{2}$ is a functional Hilbert space of holomorphic functions on $B$, with the reproducing kernel

$$
k_{q}(z, \zeta)=(1-\langle z, \zeta\rangle)^{-(n+q)} \quad(z, \zeta \in B)
$$

and with

$$
\phi_{a, q}(z)=c_{\alpha}(q) z^{\alpha} \quad\left(\alpha \in \mathbb{Z}_{+}^{n}\right)
$$

as an orthonormal basis, where

$$
c_{\alpha}(q)=\sqrt{(n+q)_{|\alpha|} / \alpha !} .
$$

Here $(a)_{m}=\Gamma(a+m) / \Gamma(a)$. The orthogonal projection of $L_{q}^{2}$ onto $A_{q}^{2}$ is denoted by $P_{q}$, and thus

$$
\left(P_{q} f\right)(z)=\left\langle f, k_{q}(\cdot, z)\right\rangle_{q}=\int f(\zeta) k_{q}(z, \zeta) d v_{q}(\zeta)
$$

We shall need the following well-known projection theorem (see Beatrous and Burbea [1]). It is due to Korányi and Vagi [9] when $q=0$ and to Forelli and Rudin [7] when $q>0$.

TheOREM 1.1. Let $q \geq 0$ and $1<p<\infty$. Then the operator $P_{q}$ is a continuous projection of $L_{q}^{p}$ onto $A_{q}^{p}$ with norm $m_{q}(p)$ satisfying $m_{q}(2)=1$ and $m_{q}(p)=m_{q}\left(p^{\prime}\right)$ where $p^{\prime}=p /(p-1)$. In particular, the dual space of $A_{q}^{p}$ is isomorphic to $A_{q}^{p^{\prime}}$, with the duality given by the $L_{q}^{2}$-pairing $\langle,\rangle_{q}$.

We shall also need the following results, whose proofs may be found in Beatrous and Burbea [1]. To some extent, these results form a motivation for this paper.

ThEOREM 1.2. Let $0<p \leq r \leq \infty$ and $Q=(n+q) / p-n / r$, and let $f \in A_{q}^{p}$. Then

$$
M_{r}(f: \rho) \leq c_{1}(1-\rho)^{-Q}\|f\|_{p, q} \quad(0<\rho<1),
$$

where $c_{1}=c_{1}(p, q, r)>0$ is a constant. If , in addition, $Q>0$ then also

$$
M_{r}(f: \rho)=o\left((1-\rho)^{-Q}\right) \quad\left(\text { as } \rho \rightarrow 1^{-}\right) .
$$


Moreover, if also $p<r$ and $p \leq k \leq \infty$ then

$$
\left\{\int_{0}^{1}(1-\rho)^{k Q-1} M_{r}^{k}(f: \rho) d \rho\right\}^{1 / k} \leq c_{2}\|f\|_{p, q}
$$

where $c_{2}=c_{2}(k, p, q, r)>0$ is a constant.

For $f \in H(B)$ with $a_{\alpha}=a_{\alpha}(f), \alpha \in \mathbb{Z}_{+}^{n}$, see (2.1) below, and $s \in \mathbb{R}$, the fractional derivative of order $s$ of $f$ is defined as the function $D^{s} f$ in $H(B)$ so that $a_{\alpha}\left(D^{s} f\right)=(1+|\alpha|)^{s} a_{\alpha}, \alpha \in \mathbb{Z}_{+}^{n}$. Let $0<p \leq \infty, q \geq 0$ and $s \in \mathbb{R}$. The space $A_{q, s}^{p}=A_{q, s}^{p}(B)$ is defined as the space of all $f \in H(B)$ so that $D^{s} f \in A_{q}^{p}$. This is a quasi-Banach space with a quasi-norm $\|\cdot\|_{p, q: s}$ given by $\|f\|_{p, q: s}=\left\|D^{s} f\right\|_{p, q}$. In particular, $A_{q, 0}^{p} \equiv A_{q}^{p}$.

Theorem 1.3. Let $0<p \leq \infty, q_{j} \geq 0$ and $s_{j} \in \mathbb{R}(j=1,2)$ such that $s_{1}-s_{2}=\left(q_{1}-q_{2}\right) / p$.

(i) If $0<p \leq \infty$ and $q_{j}>0(j=1,2)$, then $A_{q_{1}, s_{1}}^{p}=A_{q_{2}, s_{2}}^{p}$, with equivalent quasi-norms.

(ii) If $2 \leq p \leq \infty$ and $q_{2}=0$, then $A_{0, s_{2}}^{p} \subset A_{q_{1}, s_{1}}^{p}$ and the inclusion is continuous.

(iii) If $0<p \leq 2$ and $q_{2}=0$, then $A_{q_{1}, s_{1}}^{p} \subset A_{0, s_{2}}^{p}$ and the inclusion is continuous.

TheOREM 1.4. Let $0<p_{1}<p_{2}<\infty, q_{j} \geq 0$ and $s_{j} \in \mathbb{R}(j=1,2)$ such that $s_{1}-s_{2}=\left(n+q_{1}\right) / p_{1}-\left(n+q_{2}\right) / p_{2}$. Then $A_{q_{1}, s_{1}}^{p_{1}} \subset A_{q_{2}, s_{2}}^{p_{2}}$ and the inclusion is continuous.

A classical result of Hardy and Littlewood [8] states that if $f$ is in $A_{0}^{p}(\Delta)$, $0<p \leq 2$, with $a_{m}=a_{m}(f), m \in \mathbb{Z}_{+}$, then

$$
\sum_{m=0}^{\infty}(m+1)^{p-2}\left|a_{m}\right|^{p} \leq c_{p}\|f\|_{p, 0}^{p}
$$

where $c_{p}>0$ is a constant depending only on $p$. In this paper, we provide an extension of this result to $A_{q}^{p}(B), q \geq 0,0<p \leq 2$, and use this extension to study some related multiplier problems in the ball $B$.

In Section 2 we study growth estimates of functions in $A_{q}^{p}$ in terms of their Taylor coefficients and state the two main theorems (Theorems 2.1 and 2.2). We also prove two Lebesgue-Riemann type lemmas (Theorems 2.8 and 2.9). Section 3 is devoted to the proofs of Theorems 2.1 and 2.2 with some remarks on the sharpness of these theorems. This section also contains some sharp results (Theorem 3.4 and its corollaries) on growth estimates of functions in $A_{q}^{p}$ in terms of their homogeneous expansions. In Section 4 we study multiplier problems from Hardy and certain weighted Bergman spaces into spaces of the same type (Theorem 4.12) or into certain spaces of sequences (Theorems 4.2, 4.3 and 4.7). We also establish two quadratic 
inequalities of the Hardy-Hilbert type (Theorems 4.5 and 4.6) in terms of holomorphic functions with bounded mean oscillation (BMO) on $\partial B$.

2. Coefficient estimates for functions in $A_{q}^{p}$. For $\alpha=\left(\alpha_{1}, \ldots, \alpha_{n}\right)$ in $\mathbb{Z}_{+}^{n}, \partial^{\alpha}$ denotes the differential operator $\partial^{\alpha}=\partial_{1}^{\alpha_{1}} \ldots \partial_{n}^{\alpha_{n}}$ where $\partial_{j}=$ $\partial / \partial z_{j}(j=1, \ldots, n)$. Given any $f$ in $H(B)$, we consider its Taylor coefficients

$$
a_{\alpha}=a_{\alpha}(f)=\left(\partial^{\alpha} f\right)(0) / \alpha ! \quad\left(\alpha \in \mathbb{Z}_{+}^{n}\right)
$$

and thus

$$
f(z)=\sum a_{\alpha} z^{\alpha}
$$

with normal convergence in $B$. A calculation based on the orthonormal basis $\left\{\phi_{\alpha, q}\right\}$ in (1.1)-(1.2) gives the following Parseval identity:

$$
\|f\|_{2, q}^{2}=\sum \frac{\alpha !}{(n+q)_{|\alpha|}}\left|a_{\alpha}\right|^{2} \quad(q \geq 0) .
$$

The situation for $p \neq 2$ is more involved, and the main purpose of Section 3 is to prove the following two theorems which are due to Hardy and Littlewood when $n=1$ and $q=0$ (see [3, pp. 93-101]).

ThEOREM 2.1. Let $q \geq 0$ and $0<p \leq 2$. Then, for $f \in H(B)$ with $a_{\alpha}=a_{\alpha}(f), \alpha \in \mathbb{Z}_{+}^{n}$, we have

$$
\sum(|\alpha|+1)^{(n+q / 2)(p-2)}\left\{\frac{\alpha !}{(n+q)_{|\alpha|}}\right\}^{p / 2}\left|a_{\alpha}\right|^{p} \leq c\|f\|_{p, q}^{p}
$$

where $c=c(p, q)>0$ is a constant.

TheOrem 2.2. Let $q \geq 0$ and $2 \leq p<\infty$. Then, for $f \in H(B)$ with $a_{\alpha}=a_{\alpha}(f), \alpha \in \mathbb{Z}_{+}^{n}$, we have

$$
\|f\|_{p, q}^{p} \leq c \sum(|\alpha|+1)^{(n+q / 2)(p-2)}\left\{\frac{\alpha !}{(n+q)_{|\alpha|}}\right\}^{p / 2}\left|a_{\alpha}\right|^{p}
$$

where $c=c(p, q)>0$ is a constant.

For the proof of the first theorem we derive some preliminary estimates on the growth of $M_{1}(f: \rho)$ and then follow an idea of Flett [6] by utilizing Theorem 1.2. The second theorem will be obtained from the first theorem by a duality argument based on Theorem 1.1. For this purpose we start with the following lemmas, the first of which is a trivial consequence of Stirling's formula.

Lemma 2.3. For $a, b>0$ and $m \in \mathbb{Z}_{+}$, we have

$$
c_{1}(m+1)^{a-b} \leq \frac{\Gamma(m+a)}{\Gamma(m+b)} \leq c_{2}(m+1)^{a-b}
$$


where $c_{j}(j=1,2)$ is a positive constant which is independent of $m$.

LEMMA 2.4. For $0 \leq a \leq b<\infty$ and $0 \leq p \leq 1$, we have

$$
a^{p} b-a b^{p} \geq(1-p) a^{p}(b-a) .
$$

Proof. We may assume that $0<a<b$ and $0<p<1$. Then

$$
a^{p} b-a b^{p}=a^{p} b\left\{1-(a / b)^{1-p}\right\} \geq(1-p) a^{p}(b-a) .
$$

The last inequality follows from the positivity of the function $g(x)=1-$ $x^{r}-r(1-x)$ for $0<x<1$ and $0<r<1$.

Lemma 2.5. For $a_{j} \geq 0, j=1, \ldots, N$, and $0<p<\infty$, we have

$$
\sum_{j=1}^{N} a_{j}^{p} \leq N^{1-\ell}\left(\sum_{j=1}^{N} a_{j}\right)^{p}
$$

where $\ell=\min (1, p)$.

Proof. For $1 \leq p<\infty$ the inequality is trivial, while for $0<p<1$ the inequality follows from the concavity of $x^{p}, x \geq 0$.

Lemma 2.6. For $z \in \mathbb{C}^{n}, m \in \mathbb{Z}_{+}$and $0<p<\infty$, we have

$$
\sum_{|\alpha|=m}\left(\frac{m !}{\alpha !}\right)^{p / 2}\left|z^{\alpha}\right|^{p} \leq\left(\begin{array}{c}
n+m-1 \\
m
\end{array}\right)^{1-\ell}\|z\|^{p m}
$$

where $\ell=\min (1, p / 2)$.

Proof. This follows from Lemma 2.5 by observing that

$$
\sum_{|\alpha|=m} 1=\left(\begin{array}{c}
n+m-1 \\
m
\end{array}\right)
$$

and that

$$
\sum_{|\alpha|=m} \frac{m !}{\alpha !}\left|z^{\alpha}\right|^{2}=\|z\|^{2 m}
$$

Lemma 2.7. Let $f \in H(B)$ with $a_{\alpha}=a_{\alpha}(f), \alpha \in \mathbb{Z}_{+}^{n}$, and let $0<\rho<1$. Then, for $\alpha \in \mathbb{Z}_{+}^{n}$ with $|\alpha|=m$ and any $q \geq 0$, we have

$\rho^{m} \alpha ! a_{\alpha}=\frac{\Gamma(n+q+m)}{\Gamma(n) \Gamma(q)} \int_{0}^{1} r^{n+m / 2-1}(1-r)^{q-1}\left(\int_{\partial B} f(\sqrt{r} \rho z) \bar{z}^{\alpha} d v_{0}(z)\right) d r$ where for $q=0$

$$
\rho^{m} \alpha ! a_{\alpha}=\frac{\Gamma(n+m)}{\Gamma(n)} \int_{\partial B} f(\rho z) \bar{z}^{\alpha} d v_{0}(z) .
$$


Proof. For $q \geq 0$, we let $\phi_{\alpha}=\phi_{a, q}$ and $c_{\alpha}=c_{\alpha}(q)$. Then by (1.1)-(1.2),

$$
c_{\alpha}^{-2} \rho^{|\alpha|} a_{\alpha}=c_{\alpha}^{-1}\left\langle f_{\rho}, \phi_{\alpha}\right\rangle_{q}=\int f(\rho z) \bar{z}^{\alpha} d v_{q}(z) .
$$

The result for $q=0$ follows now at once. For $q>0$, we use polar coordinates to obtain

$$
\begin{aligned}
& \rho^{m} \alpha ! a_{\alpha}=(n+q)_{m} \int f(\rho z) \bar{z}^{\alpha} d v_{q}(z) \\
& =(n+q)_{m}(q)_{n} 2 \int_{0}^{1} r^{2 n+m-1}\left(1-r^{2}\right)^{q-1}\left(\int_{\partial B} f(r \rho z) \bar{z}^{\alpha} d v_{0}(z)\right) d r \\
& =(n+q)_{m}(q)_{n} \int_{0}^{1} r^{n+m / 2-1}(1-r)^{q-1}\left(\int_{\partial B} f(\sqrt{r} \rho z) \bar{z}^{\alpha} d v_{0}(z)\right) d r,
\end{aligned}
$$

and the result follows.

Before proceeding with the proofs of Theorems 2.1 and 2.2, we shall state and prove the following two results which are of interest on their own right:

TheOREM 2.8. Let $q \geq 0,0<p \leq 1$ and $f \in A_{q}^{p}$, and let $a_{\alpha}=a_{\alpha}(f)$, $\alpha \in \mathbb{Z}_{+}^{n}$. Then for $\alpha \in \mathbb{Z}_{+}^{n}$ with $|\alpha|=m$ we have

$$
\left|a_{\alpha}\right| / c_{\alpha}^{2}(q) \leq c(m+1)^{(n+q)(1 / p-1)}\|f\|_{p, q}
$$

and

$$
a_{\alpha} / c_{\alpha}^{2}(q)=o\left((m+1)^{(n+q)(1 / p-1)}\right) \quad(\text { as } m \rightarrow \infty)
$$

where $c=c(p, q)>0$ is a constant.

Proof. By Lemma 2.7

$$
\begin{aligned}
& \left|a_{\alpha}\right| / c_{\alpha}^{2}(q) \\
& \leq \rho^{-m} \frac{\Gamma(n+q)}{\Gamma(n) \Gamma(q)} \int_{0}^{1} r^{n+m / 2-1}(1-r)^{q-1}\left(\int_{\partial B}|f(\sqrt{r} \rho z)|\left|z^{\alpha}\right| d v_{0}(z)\right) d r \\
& \leq \rho^{-m} M_{1}(f: \rho) \frac{\Gamma(n+q) \Gamma(n+m / 2)}{\Gamma(n) \Gamma(n+q+m / 2)},
\end{aligned}
$$

and thus by Lemma 2.3 ,

$$
\left|a_{\alpha}\right| / c_{\alpha}^{2}(q) \leq c_{2}(m+1)^{-q} \rho^{-m}(1-\rho)^{n-(n+q) / p}\|f\|_{p, q} \quad(0<\rho<1)
$$

where $c_{j}=c_{j}(p, q)>0, j=1,2$, are constants. Thus, by choosing $\rho=$ $1-(m+1)^{-1}$, the first part of the theorem follows. Similarly, using Theorem 1.2 , the second part of the theorem follows, provided $0<p<1$. In the case $p=1$, we have

$$
a_{\alpha}\left(1-\rho^{|\alpha|}\right)=c_{\alpha}(q)\left\langle f-f_{\rho}, \phi_{\alpha, q}\right\rangle_{q}
$$


and thus

$$
\left|a_{\alpha}\right|\left(1-\rho^{|\alpha|}\right) \leq c_{\alpha}(q)\left\|f-f_{\rho}\right\|_{1, q}\left\|\phi_{\alpha, q}\right\|_{\infty, q} \leq c_{\alpha}^{2}(q)\left\|f-f_{\rho}\right\|_{1, q} .
$$

It follows that

$$
\left|a_{\alpha}\right| / c_{\alpha}^{2}(q) \leq\left(1-\rho^{m}\right)^{-1}\left\|f-f_{\rho}\right\|_{1, q} \quad(0<\rho<1) .
$$

Again, we choose $\rho=1-(m+1)^{-1}$, and observe that in this case we have $\lim _{m \rightarrow \infty}\left(1-\rho^{m}\right)^{-1}=e /(e-1)$ and $\lim _{m \rightarrow \infty}\left\|f-f_{\rho}\right\|_{1, q}=0$. This shows that $a_{\alpha} / c_{\alpha}^{2}(q)=o(1)$ if $f \in A_{q}^{1}$, and the proof is complete.

TheOREM 2.9. Let $q \geq 0,0<p<\infty$ and $0<\rho<1$, and let $f \in H(B)$ with $a_{\alpha}=a_{\alpha}(f), \alpha \in \mathbb{Z}_{+}^{n}$. Then, for any $m \in \mathbb{Z}_{+}$, we have

$$
M_{1}^{p}(f: \rho) \geq c \rho^{p m}(m+1)^{\ell(1-n)+p q / 2} \sum_{|\alpha|=m}\left\{\frac{\alpha !}{(n+q)_{|\alpha|}}\right\}^{p / 2}\left|a_{\alpha}\right|^{p}
$$

where $\ell=\max (1, p)$ and $c=c(p, q)>0$ is a constant.

Proof. By Lemma 2.7, we have for $\alpha \in \mathbb{Z}_{+}^{n}$ with $|\alpha|=m$,

$$
\begin{aligned}
& \rho^{m} \alpha !\left|a_{\alpha}\right| \\
& \leq \frac{\Gamma(n+q+m)}{\Gamma(n) \Gamma(q)} \int_{0}^{1} r^{n+m / 2-1}(1-r)^{q-1}\left(\int_{\partial B}|f(\sqrt{r} \rho z)|\left|z^{\alpha}\right| d v_{0}(z)\right) d r .
\end{aligned}
$$

Upon multiplying both sides of this inequality by $(m ! / \alpha !)^{1 / 2}$ and summing over all indices $\alpha$ with $|\alpha|=m$, we obtain, using Lemma 2.6 with $p=1$,

$$
\begin{aligned}
& \rho^{m} \sum_{|\alpha|=m}(m ! \alpha !)^{1 / 2}\left|a_{\alpha}\right| \\
& \leq \frac{\Gamma(n+q+m)}{\Gamma(n) \Gamma(q)}\left(\begin{array}{c}
n+m-1 \\
m
\end{array}\right)^{1 / 2} M_{1}(f: \rho) \int_{0}^{1} r^{n+m / 2-1}(1-r)^{q-1} d r \\
& =\frac{\Gamma(n+m / 2) \Gamma(n+q+m)}{\Gamma(n) \Gamma(n+q+m / 2)}\left(\begin{array}{c}
n+m-1 \\
m
\end{array}\right)^{1 / 2} M_{1}(f: \rho) .
\end{aligned}
$$

It follows, using Lemma 2.5 with $N=\sum_{|\alpha|=m} 1=\left(\begin{array}{c}n+m-1 \\ m\end{array}\right)$, that

$$
\begin{aligned}
& \rho^{p m} \sum_{|\alpha|=m}(m ! \alpha !)^{p / 2}\left|a_{\alpha}\right|^{p} \\
& \leq\left(\begin{array}{c}
n+m-1 \\
m
\end{array}\right)^{1-k+p / 2}\left(\frac{\Gamma(n+m / 2) \Gamma(n+q+m)}{\Gamma(n) \Gamma(n+q+m / 2)}\right)^{p} M_{1}^{p}(f: \rho)
\end{aligned}
$$


where $k=\min (1, p)$. Therefore, by Lemma 2.3 ,

$$
\begin{aligned}
& M_{1}^{p}(f: \rho) \\
& \geq \frac{\{\Gamma(n)\}^{3 p / 2+1-k}}{\{\Gamma(n+q)\}^{p / 2}} \rho^{p m}\left(\frac{\Gamma(1+m)}{\Gamma(n+m)}\right)^{1-k+p / 2}\left(\frac{\Gamma(n+q+m / 2)}{\Gamma(n+m / 2)}\right)^{p} \\
& \quad \times\left(\frac{\Gamma(1+m)}{\Gamma(n+q+m)}\right)^{p} \sum_{|\alpha|=m}\left\{\frac{\alpha !}{(n+q)_{|\alpha|}}\right\}^{p / 2}\left|a_{\alpha}\right|^{p} \\
& \geq c \rho^{p m}(m+1)^{\ell(1-n)+p q / 2} \sum_{|\alpha|=m}\left\{\frac{\alpha !}{(n+q)_{|\alpha|}}\right\}^{p / 2}\left|a_{\alpha}\right|^{p},
\end{aligned}
$$

and the proof is complete.

3. Proofs of Theorems 2.1 and 2.2, and sharpness. We now prove Theorem 2.1. We first assume that $0<p<1$. Then, by Theorem 1.2, with a constant $c_{1}=c_{1}(p, q)>0$, we have

$$
\begin{aligned}
c_{1}\|f\|_{p, q}^{p} & \geq \int_{0}^{1}(1-\rho)^{n+q-p n-1} M_{1}^{p}(f: \rho) d \rho \\
& =\sum_{m=0}^{\infty} \int_{s_{m}}^{s_{m+1}}(1-\rho)^{n+q-p n-1} M_{1}^{p}(f: \rho) d \rho \\
& \geq \sum_{m=0}^{\infty} M_{1}^{p}\left(f: s_{m}\right) \int_{s_{m}}^{s_{m}+1}(1-\rho)^{n+q-p n-1} d \rho \\
& =\frac{1}{n+q-p n} \sum_{m=0}^{\infty} M_{1}^{p}\left(f: s_{m}\right)\left\{r_{m}^{n+q-p n}-r_{m+1}^{n+q-p n}\right\},
\end{aligned}
$$

where $r_{m}=1-s_{m}(m=0,1, \ldots)$ and $\left\{s_{m}\right\}$ is any increasing sequence with $s_{0}=0$ and $\lim _{m \rightarrow \infty} s_{m}=1$. Now, by Lemma 2.4,

$$
\begin{aligned}
& r_{m}^{n+q-p n}-r_{m+1}^{n+q-p n}=\left(r_{m} r_{m+1}\right)^{n+q}\left\{r_{m}^{-n p} r_{m+1}^{-(n+q)}-r_{m}^{-(n+q)} r_{m+1}^{-n p}\right\} \\
& \quad \geq r_{m}^{n} r_{m+1}^{n+q}\left\{r_{m}^{-n p} r_{m+1}^{-n}-r_{m}^{-n} r_{m p}^{-n p}\right\} \geq(1-p) r_{m}^{n-n p} r_{m+1}^{n+q}\left(r_{m+1}^{-n}-r_{m}^{-n}\right) \\
& \quad=(1-p) r_{m}^{n-n p} r_{m+1}^{q}\left\{1-\left(r_{m}^{-1} r_{m+1}\right)^{n}\right\} \geq(1-p) r_{m}^{n-n p} r_{m+1}^{q}\left(1-r_{m}^{-1} r_{m+1}\right) \\
& \quad=(1-p) r_{m}^{n-n p} r_{m+1}^{q+1}\left(r_{m+1}^{-1}-r_{m}^{-1}\right),
\end{aligned}
$$

and therefore

$$
c_{1}\|f\|_{p, q}^{p} \geq \frac{1-p}{n+q-p n} \sum_{m=0}^{\infty} M_{1}^{p}\left(f: s_{m}\right) r_{m}^{n-n p} r_{m+1}^{q+1}\left(r_{m+1}^{-1}-r_{m}^{-1}\right) .
$$

We now specify $s_{m}=1-(m+1)^{-1}$ and so $r_{m}^{-1}=m+1$ for $m=0,1, \ldots$ Thus, 
it follows from Theorem 2.9, together with the facts that $(m+1) /(m+2) \rightarrow 1$ and $\left(1-(m+1)^{-1}\right)^{p m} \rightarrow e^{-p}$ as $m \rightarrow \infty$, that

$$
\begin{aligned}
c\|f\|_{p, q}^{p} & \geq \sum_{m=0}^{\infty}(m+1)^{(n+q / 2)(p-2)} \sum_{|\alpha|=m}\left\{\frac{\alpha !}{(n+q)_{|\alpha|}}\right\}^{p / 2}\left|a_{\alpha}\right|^{p} \\
& =\sum(|\alpha|+1)^{(n+q / 2)(p-2)}\left\{\frac{\alpha !}{(n+q)_{|\alpha|}}\right\}^{p / 2}\left|a_{\alpha}\right|^{p} .
\end{aligned}
$$

This gives the desired result for $0<p<1$. For $p=2$, the result is trivially true by virtue of Parseval identity (2.2). In order to obtain the full result for $0<p \leq 2$, we use the complex interpolation method as the one found in Stein and Weiss [10].

We consider the space of sequences $\ell_{q}^{p}=\ell_{q}^{p}\left(\mathbb{Z}_{+}^{n}\right)$, defined as the $L^{p}$-space of functions on $\mathbb{Z}_{+}^{n}$ with respect to the counting measure $d \nu_{q}$, given by

$$
\nu_{q}(\alpha)=(|\alpha|+1)^{-2(n+q / 2)} \quad\left(\alpha \in \mathbb{Z}_{+}^{n}\right) .
$$

Thus, in particular, any sequence element $\underline{a}=\left(a_{\alpha}\right), \alpha \in \mathbb{Z}_{+}^{n}$, in $\ell_{q}^{p}$ has the quasi-norm

$$
\|\underline{a}\|_{\ell_{q}^{p}}=\left\{\sum(|\alpha|+1)^{-2(n+q / 2)}\left|a_{\alpha}\right|^{p}\right\}^{1 / p} .
$$

For each $f \in H(B)$ with $a_{\alpha}=a_{\alpha}(f), \alpha \in \mathbb{Z}_{+}^{n}$, we define

$$
(T f)(\alpha)=(|\alpha|+1)^{n+q / 2}\left\{\frac{\alpha !}{(n+q)_{|\alpha|}}\right\}^{1 / 2} a_{\alpha},
$$

and thus $T$ is a linear operator from $H(B)$ into the space of sequences on $\mathbb{Z}_{+}^{n}$. Moreover, by what we have shown before, $T$ is a bounded operator from $A_{q}^{p}$ into $\ell_{q}^{p}, 0<p<1$, with quasi-norm $\leq c^{1 / p}$ and from $A_{q}^{2}$ into $\ell_{q}^{2}$ with norm 1. It follows from the complex interpolation method that $T$ is a bounded operator from $A_{q}^{p}$ into $\ell_{q}^{p}, 0<p \leq 2$ with quasi-norm $\leq c^{1 / p}$, where $c=c(p, q)>0$ is a constant. This means that $\|T f\|_{\ell_{q}^{p}}^{p} \leq c\|f\|_{p, q}^{p}$ for every $f \in A_{q}^{p}(0<p \leq 2, q \geq 0)$, which is a reformulation of Theorem 2.1, and this concludes its proof.

To prove Theorem 2.2, we let $p^{\prime}=p /(p-1)$ for $2 \leq p<\infty$ and consider the orthogonal projection $P_{q}$ of $L_{q}^{2}$ onto $A_{q}^{2}, q \geq 0$. Let $g \in L_{q}^{p^{\prime}}$ with $\|g\|_{p^{\prime}, q}=1$. By Theorem 1.1, $P_{q} g$ is in $A_{q}^{p^{\prime}}$ with $\left\|P_{q} g\right\|_{p^{\prime}, q} \leq m_{q}(p)$, and so we let $b_{\alpha}=a_{\alpha}\left(P_{q} g\right)$ for $\alpha \in \mathbb{Z}_{+}^{n}$. Let $f \in H(B)$ with $a_{\alpha}=a_{\alpha}(f)$ for $\alpha \in \mathbb{Z}_{+}^{n}$, and consider the polynomials

$$
F_{m}(z)=\sum_{|\alpha| \leq m} a_{\alpha} z^{\alpha} \quad(m=0,1, \ldots) .
$$


By the self-adjointness of $P_{q}$, we find that

$$
\left\langle g, F_{m}\right\rangle_{q}=\left\langle g, P_{q} F_{m}\right\rangle_{q}=\left\langle P_{q} g, F_{m}\right\rangle_{q},
$$

and thus

$$
\left\langle g, F_{m}\right\rangle_{q}=\sum_{|\alpha| \leq m} c_{\alpha}^{-2}(q) b_{\alpha} \bar{a}_{\alpha}
$$

It follows from the Hölder inequality that

$$
\begin{aligned}
\left|\left\langle g, F_{m}\right\rangle_{q}\right| \leq & \sum_{|\alpha| \leq m} c_{\alpha}^{-2}(q)\left|b_{\alpha} a_{\alpha}\right| \\
= & \sum_{|\alpha| \leq m}(|\alpha|+1)^{(n+q / 2)\left(2-2 / p-2 / p^{\prime}\right)} c_{\alpha}^{-2}(q)\left|b_{\alpha} a_{\alpha}\right| \\
\leq & \left\{\sum_{|\alpha| \leq m}(|\alpha|+1)^{(n+q / 2)\left(p^{\prime}-2\right)} c_{\alpha}^{-p^{\prime}}(q)\left|b_{\alpha}\right|^{p^{\prime}}\right\}^{1 / p^{\prime}} \\
& \times\left\{\sum_{|\alpha| \leq m}(|\alpha|+1)^{(n+q / 2)(p-2)} c_{\alpha}^{-p}(q)\left|a_{\alpha}\right|^{p}\right\}^{1 / p} .
\end{aligned}
$$

Since $1<p^{\prime} \leq 2$, we may apply Theorem 2.1 to $P_{q} g$, and therefore obtain

$$
\left|\left\langle g, F_{m}\right\rangle_{q}\right| \leq c_{1} m_{q}(p)\left\{\sum_{|a| \leq m}(|\alpha|+1)^{(n+q / 2)(p-2)} c_{\alpha}^{-p}(q)\left|a_{\alpha}\right|^{p}\right\}^{1 / p}
$$

where $c_{1}=c_{1}(p, q)>0$. It follows that

$$
\left|\left\langle g, F_{m}\right\rangle_{q}\right| \leq c^{1 / p}\left\{\sum_{|\alpha| \leq m}(|a|+1)^{(n+q / 2)(p-2)}\left\{\frac{\alpha !}{(n+q)_{|\alpha|}}\right\}^{p / 2}\left|a_{\alpha}\right|^{p}\right\}^{1 / p},
$$

and hence, taking the supremum over all $g \in L_{q}^{p^{\prime}}$ with $\|g\|_{p^{\prime}, q}=1$,

$$
\left\|F_{m}\right\|_{p, q}^{p} \leq c \sum(|a|+1)^{(n+q / 2)(p-2)}\left\{\frac{\alpha !}{(n+q)_{|\alpha|}}\right\}^{p / 2}\left|a_{\alpha}\right|^{p} .
$$

The result now follows by letting $m \rightarrow \infty$.

We conclude these proofs by making a few remarks about the "sharpness" of Theorems 2.1 and 2.2. It is clear from (2.2) that the exponent in the series is best possible when $p=2$. For $n=1$ and $q=0$, this exponent is best possible even when $p \neq 2$ (see [3, p. 106]). In fact, consider the holomorphic function

$$
g_{s}(z)=\left(1-\left\langle z, \zeta_{0}\right\rangle\right)^{-s} \quad\left(s>0, \zeta_{0} \in \partial B\right)
$$

on $B$. It follows that

$$
g_{s}(z)=\sum \frac{(s)_{|\alpha|}}{\alpha !} \bar{\zeta}_{0} \alpha_{z} \alpha
$$


and thus

$$
\left\|g_{s}\right\|_{p, q}^{p}=\sum_{m=0}^{\infty} \frac{\left\{(s p / 2)_{m}\right\}^{2}}{m !(n+q)_{m}} \quad(0<p<\infty, q \geq 0) .
$$

The last series, by virtue of Lemma 2.3 , is equivalent to $\sum(m+1)^{s p-n-q-1}$, and thus $g_{s} \in A_{q}^{p}$ if and only if $s<(n+q) / p(q \geq 0,0<p<\infty)$. On the other hand, the series corresponding to that of Theorem 2.1 for the coefficients of $g_{s}$ is

$$
\begin{aligned}
& \sum(|\alpha|+1)^{(n+q / 2)(p-2)}\left\{\frac{\alpha !}{(n+q)_{|\alpha|}}\right\}^{p / 2}\left\{\frac{\left.(s)_{|\alpha|}\left|\zeta_{0}^{\alpha}\right|\right\}^{p}}{\alpha !}\left\{\frac{(s)_{m}}{(n+q)_{m}} \cdot \frac{(s)_{m}}{m !}\right\}^{p / 2} \sum_{|\alpha|=m}\left(\frac{m !}{\alpha !}\right)^{p / 2}\left|\zeta_{0}^{\alpha}\right|^{p} .\right.
\end{aligned}
$$

If $n=1$, the last series, again by virtue of Lemma 2.3 , is equivalent to that in (3.1), and therefore the exponent in the series of Theorems 2.1 and 2.2 is best possible for $n=1, q \geq 0$ and $p \neq 2$; on the other hand, the last series, by virtue of Lemma 2.6 , is dominated by

$$
\sum_{m=0}^{\infty}(m+1)^{(n+q / 2)(p-2)}\left\{\frac{(s)_{m}}{(n+q)_{m}} \cdot \frac{(s)_{m}}{m !}\right\}^{p / 2}\left(\begin{array}{c}
n+m-1 \\
m
\end{array}\right)^{1-\ell}
$$

where $\ell=\min (1, p / 2)$. The last series is therefore equivalent to that in $(3.1)$ if $p<2$. This, unfortunately, does not show that the exponent in the series of Theorem 2.1 is best possible for $n>1, q \geq 0$ and $0<p<2$, and thus this question remains open.

The problem of "sharpness" of Theorems 2.1 and 2.2 lies, of course, with the estimate given in Theorem 2.9. In the one variable case $(n=1)$, this estimate is sufficient to yield sharpness. In the several variable case $(n>1)$, however, a sharper inequality which involves all the Taylor coefficients of certain weight is probably required. At this stage the specific form of such inequality is not known, but we content ourselves with some sharp results where the Taylor coefficients $a_{\alpha}=a_{\alpha}(f), \alpha \in \mathbb{Z}_{+}^{n}$, of $f \in H(B)$ are replaced by

$$
F_{m}(z)=\sum_{|\alpha|=m} a_{\alpha} z^{\alpha}
$$

i.e. $F_{m}$ is a homogeneous polynomial of degree $m(m=0,1, \ldots)$. Accordingly, $f(z)=\sum_{m=0}^{\infty} F_{m}(z)$ is the homogeneous expansion of $f$ at 0 .

Before proceeding, we introduce some further notation. For $z=$ $\left(z_{1}, \ldots, z_{n}\right)$ and $\zeta=\left(\zeta_{1}, \ldots, \zeta_{n}\right)$ in $\mathbb{C}^{n}$ we let $z \cdot \zeta$ denote the point $\left(z_{1} \zeta_{1}, \ldots, z_{n} \zeta_{n}\right)$ in $\mathbb{C}^{n}$. For a function $f$ on $B$, and $\zeta \in \bar{\Delta}^{n}$, we define $f_{\zeta}(z)=f(z \cdot \zeta), z \in B$. It is then obvious that if $f \in H(B)$ then $f_{\zeta} \in H(B)$ 
for every $\zeta \in \bar{\Delta}^{n}$. For $q>0, d \nu_{q}$ denotes the probability measure

$$
d \nu_{q}(r)=\frac{\Gamma(n+q)}{\Gamma(n) \Gamma(q)} r^{n-1}(1-r)^{q-1} d r
$$

on $(0,1)$. Observe that for $0<p \leq \infty, q>0$ and $f \in H(B)$ we have the identity

$$
\|f\|_{p, q}=\left\{\int_{0}^{1} M_{q}^{p}(f: \sqrt{r}) d \nu_{q}(r)\right\}^{1 / p},
$$

which is also correct when $q=0$, in the sense that $\lim _{q \rightarrow 0^{+}}\|f\|_{p, q}=\|f\|_{p, 0}$.

Lemma 3.1 Let $0<p \leq \infty, q \geq 0$ and $\alpha=\left(\alpha_{1}, \ldots, \alpha_{n}\right) \in \mathbb{Z}_{+}^{n}$. Then

$$
\left\|z^{\alpha}\right\|_{p, q}^{p}=\frac{\Gamma(n+q)}{\Gamma(n+q+p|\alpha| / 2)} \prod_{j=1}^{n} \Gamma\left(p \alpha_{j} / 2+1\right) .
$$

Pro of. We may assume that $0<p<\infty$, and we consider the integral

$$
I_{p}(\alpha)=\int_{\mathbb{C}^{n}}\left|z^{\alpha}\right|^{p} e^{-\|z\|^{2}} d v_{1}(z) .
$$

It follows that

$$
\begin{aligned}
I_{p}(\alpha) & =\int_{0}^{\infty} e^{-r} r^{p|\alpha| / 2}\left(\int_{\partial B}\left|z^{\alpha}\right|^{p} d \sigma(z)\right) d \nu_{1}(r) \\
& =n\left\|z^{\alpha}\right\|_{p, 0}^{p} \int_{0}^{\infty} e^{-r} r^{p|\alpha| / 2+n-1} d r=n \Gamma(n+p|\alpha| / 2)\left\|z^{\alpha}\right\|_{p, 0}^{p} .
\end{aligned}
$$

On the other hand, we also have

$$
I_{p}(\alpha)=n ! \prod_{j=1}^{n} \int_{\mathbb{C}}|\lambda|^{p \alpha_{j} / 2} e^{-|\lambda|^{2}} d A(\lambda)
$$

where $d A(\lambda)=i(d \lambda \wedge d \bar{\lambda}) /(2 \pi), \lambda \in \mathbb{C}$. Therefore

$$
I_{p}(\alpha)=n ! \prod_{j=1}^{n} \int_{0}^{\infty} e^{-r} r^{p \alpha_{j} / 2} d r=n ! \prod_{j=1}^{n} \Gamma\left(p \alpha_{j} / 2+1\right),
$$

and

$$
\left\|z^{\alpha}\right\|_{p, 0}^{p}=\frac{\Gamma(n)}{\Gamma(n+p|\alpha| / 2)} \prod_{j=1}^{n} \Gamma\left(p \alpha_{j} / 2+1\right) .
$$


This proves the lemma for $q=0$. For $q>0$, we deduce that

$$
\begin{aligned}
\left\|z^{\alpha}\right\|_{p, q}^{p} & =\int_{0}^{1} r^{|\alpha| p / 2}\left\|z^{\alpha}\right\|_{p, 0}^{p} d \nu_{q}(r) \\
& =\left\|z^{\alpha}\right\|_{p, 0}^{p} \frac{\Gamma(n+q)}{\Gamma(n) \Gamma(q)} \int_{0}^{1} r^{|\alpha| p / 2+n-1}(1-r)^{q-1} d r \\
& =\left\|z^{\alpha}\right\|_{p, 0}^{p} \frac{\Gamma(n+q) \Gamma(n+p|\alpha| / 2)}{\Gamma(n) \Gamma(n+q+p|\alpha| / 2)} \\
& =\frac{\Gamma(n+q)}{\Gamma(n+q+p|\alpha| / 2)} \prod_{j=1}^{n} \Gamma\left(p \alpha_{j} / 2+1\right),
\end{aligned}
$$

and the proof is complete.

LEMMA 3.2. Let $0<p \leq \infty, q \geq 0, f \in H(B)$ and $\zeta \in \bar{\Delta}^{n}$. Then $\left\|f_{\zeta}\right\|_{p, q} \leq\|f\|_{p, q}$.

Proof. The case $p=\infty$ is trivial, and so we assume that $0<p<\infty$. Let $\zeta=\left(r_{1} e^{i \theta_{1}}, \ldots, r_{n} e^{i \theta_{n}}\right), \theta_{j} \in \mathbb{R}, r_{j} \geq 0$ with $r=\max \left(r_{1}, \ldots, r_{n}\right) \leq 1$. It follows from the subharmonicity of $|f|^{p}$ on $B$ that

$$
\begin{aligned}
\left\|f_{\zeta}\right\|_{p, q}^{p} & =\int|f(\zeta \cdot z)|^{p} d v_{q}(z)=\int\left|f\left(r_{1} z_{1} e^{i \theta_{1}}, \ldots, r_{n} z_{n} e^{i \theta_{n}}\right)\right|^{p} d v_{q}(z) \\
& =\int\left|f\left(r_{1} z_{1}, \ldots, r_{n} z_{n}\right)\right|^{p} d v_{q}(z) \\
& =\int_{0}^{1}\left(\int_{\partial B}\left|f\left(\sqrt{\rho} r_{1} z_{1}, \ldots, \sqrt{\rho} r_{n} z_{n}\right)\right|^{p} d \sigma(z)\right) d \nu_{q}(\rho) \\
& \leq \int_{0}^{1}\left(\int_{\partial B}|f(\sqrt{\rho} z)|^{p} d \sigma(z)\right) d \nu_{q}(\rho)=\|f\|_{p, q}^{p} .
\end{aligned}
$$

This concludes the proof.

Corollary 3.3. Let $0<p \leq \infty, q \geq 0$ and $f \in H(\bar{B})$. Assume also that $f$ is homogeneous of degree $\kappa \geq 0$, i.e. $f(\lambda z)=\lambda^{\kappa} f(z)$ for every $z \in B$ and every $\lambda \in \bar{\Delta}$. Then

$$
\|f\|_{\infty} \equiv\|f\|_{\infty, q} \leq\left\{\frac{\Gamma(n+q+p \kappa / 2)}{\Gamma(n+q) \Gamma(p \kappa / 2+1)}\right\}^{1 / p}\|f\|_{p, q} .
$$

Proof. For any unitary transformation $U$ of $\mathbb{C}^{n}$, we also have that $f \circ U \in H(\bar{B})$ and that $f \circ U$ is homogeneous of degree $\kappa$. In particular, we may, without loss of generality, assume that $\|f\|_{\infty}=|f(1,0, \ldots, 0)|$. It 
follows from Lemma 3.2, with $\zeta=(1,0, \ldots, 0)$, and Lemma 3.1, that

$$
\begin{aligned}
\|f\|_{p, q} & \geq\left\|f_{\zeta}\right\|_{p, q}^{p}=\int\left|f\left(z_{1}, 0, \ldots, 0\right)\right|^{p} d v_{q}(z) \\
& =|f(1,0, \ldots, 0)|^{p} \int\left|z_{1}\right|^{\kappa p} d v_{q}(z) \\
& =\|f\|_{\infty}^{p} \frac{\Gamma(n+q)}{\Gamma(n+q+p \kappa / 2)} \Gamma(p \kappa / 2+1),
\end{aligned}
$$

which is the desired result.

We now recall the previously mentioned classical result of Hardy and Littlewood [8], namely

$$
\sum_{m=0}^{\infty}(m+1)^{p-2}\left|b_{m}\right|^{p} \leq c_{p}\|g\|_{p, q}^{p}, \quad 0<p \leq 2,
$$

and

$$
\sum_{m=0}^{\infty}(m+1)^{p-2}\left|b_{m}\right|^{p} \geq d_{p}\|g\|_{p, 0}^{p}, \quad 2 \leq p<\infty
$$

for any $g \in H(\Delta)$ with $b_{m}=a_{m}(g), m \in \mathbb{Z}_{+}^{n}$. Here the positive constants $c_{p}$ and $d_{p}$ depend only on $p$ with $c_{1}=\pi$ (see, for example, [3, p. 48]) and, of course, $c_{2}=d_{2}=1$. We now prove:

Theorem 3.4. Let $f \in H(B)$, and let $f=\sum_{m=0}^{\infty} F_{m}$ be its homogeneous expansion at 0 , where $F_{m}$ is a homogeneous polynomial of degree $m(m=$ $0,1, \ldots)$. Let $q \geq 0$. Then

$$
\begin{array}{ll}
\sum_{m=0}^{\infty}(m+1)^{p-2}\left\|F_{m}\right\|^{p} \leq c_{p}\|f\|_{p, q}^{p}, & 0<p \leq 2, \\
\sum_{m=0}^{\infty}(m+1)^{p-2}\left\|F_{m}\right\|_{p, q}^{p} \geq d_{p}\|f\|_{p, q}^{p}, & 2 \leq p<\infty,
\end{array}
$$

and the exponent in both series is sharp.

Proof. We first fix $z \in \partial B$. Then for $\lambda \in \Delta$ we have $f(\lambda z)=$ $\sum_{m=0}^{\infty} \lambda^{m} F_{m}(z)$, and thus for $0<r<1$,

$$
\sum_{m=0}^{\infty}(m+1)^{p-2}\left|F_{m}(\sqrt{r} z)\right|^{p} \leq c_{p} \frac{1}{2 \pi} \int_{0}^{2 \pi}\left|f\left(\sqrt{r} e^{i \theta} z\right)\right|^{p} d \theta, \quad 0<p \leq 2,
$$

and

$$
\sum_{m=0}^{\infty}(m+1)^{p-2}\left|F_{m}(\sqrt{r} z)\right|^{p} \geq d_{p} \frac{1}{2 \pi} \int_{0}^{2 \pi}\left|f\left(\sqrt{r} e^{i \theta} z\right)\right|^{p} d \theta, \quad 2 \leq p<\infty .
$$


Integrating both sides of the inequalities with respect to $d \sigma(z), z \in \partial B$, we obtain

$$
\begin{array}{ll}
\sum_{m=0}^{\infty}(m+1)^{p-2} M_{p}^{p}\left(F_{m}: \sqrt{r}\right) \leq c_{p} M_{p}^{p}(f: \sqrt{r}), & 0<p \leq 2, \\
\sum_{m=0}^{\infty}(m+1)^{p-2} M_{p}^{p}\left(F_{m}: \sqrt{r}\right) \geq d_{p} M_{p}^{p}(f: \sqrt{r}), & 2 \leq p<\infty .
\end{array}
$$

The desired result for $q=0$ is obtained from these by letting $r \rightarrow 1$. For $q>0$, the desired result is obtained by integrating both sides of these inequalities with respect to the probability measure $d \nu_{q}(r), 0<r<1$.

To show sharpness, we let, once again, $f=g_{s}$, i.e.

$$
f(z)=\left(1-\left\langle z, \zeta_{0}\right\rangle\right)^{-s} \quad\left(s>0, \zeta_{0} \in \partial B\right)
$$

and thus $f=\sum_{m=0}^{\infty} F_{m}$ with

$$
F_{m}(z)=\frac{(s)_{m}}{m !}\left\langle z, \zeta_{0}\right\rangle^{m} \quad(m=0,1, \ldots) .
$$

Let $0<p<\infty$. By a unitary change of variable, and by Lemma 3.1,

$$
\begin{aligned}
\int\left|\left\langle z, \zeta_{0}\right\rangle\right|^{m p} d v_{q}(z) & =\int\left|z_{1}\right|^{m p} d v_{q}(z)=\frac{\Gamma(n+q) \Gamma(m p / 2+1)}{\Gamma(m p / 2+n+q)} \\
& \simeq \sum_{m=0}^{\infty}(m+1)^{s p-n-q-1}
\end{aligned}
$$

On the other hand, by (3.1) and Lemma 2.3,

$$
\|f\|_{p, q}^{p}=\sum_{m=0}^{\infty} \frac{\left\{(s p / 2)_{m}\right\}^{2}}{m !(n+q)_{m}} \simeq \sum_{m=0}^{\infty}(m+1)^{s p-n-q-1}
$$

and the proof is complete.

Corollary 3.5. Let $f \in H(B)$, and let $f=\sum F_{m}$ be its homogeneous expansion at 0 , and let $q \geq 0$. Then for $0<p \leq 2$

$$
\begin{gathered}
\Gamma(n+q) \sum_{m=0}^{\infty}(m+1)^{p-2} \frac{\Gamma(m p / 2+1)}{\Gamma(m p / 2+n+q)}\left\|F_{m}\right\|_{\infty}^{p} \\
\leq \sum_{m=0}^{\infty}(m+1)^{p-2}\left\|F_{m}\right\|_{p, q}^{p} \leq c_{p}\|f\|_{p, q}^{p},
\end{gathered}
$$

and the exponent in both series is sharp.

Proof. The inequalities are obtained from Theorem 3.4 and Corollary 3.3. In view of Theorem 3.4 and its proof of the sharpness part, to show 
sharpness it is sufficient to show that for $f$ as given in (3.2)-(3.3) we have

$$
\sum_{m=0}^{\infty}(m+1)^{p-2} \frac{\Gamma(m p / 2+1)}{\Gamma(m p / 2+n+q)}\left\|F_{m}\right\|_{\infty}^{p} \simeq \sum_{m=0}^{\infty}(m+1)^{s p-n-q-1} .
$$

Indeed, the left hand side of this equivalence is, by Lemma 2.3,

$$
\sum_{m=0}^{\infty}(m+1)^{p-2} \frac{\Gamma(m p / 2+1)}{\Gamma(m p / 2+n+q)}\left\{\frac{(s)_{m}}{m !}\right\}^{p} \simeq \sum_{m=0}^{\infty}(m+1)^{s p-n-q-1},
$$

concluding the proof.

Corollary 3.6. Let $q \geq 0$ and $0<p \leq 2$. There exists a constant $C_{p}=C_{p}(q, n)>0$ such that for any $f \in H(B)$, with its homogeneous expansion $f=\sum F_{m}$, we have

$$
\sum_{m=0}^{\infty}(m+1)^{p-1-n-q}\left\|F_{m}\right\|_{\infty}^{p} \leq C_{p}\|f\|_{p, q}^{p} .
$$

Moreover, the exponent of the series is sharp.

Pr o of. This follows from Corollary 3.5 and Lemma 2.3.

Corollary 3.7. Let $q \geq 0$ and $f \in H(B)$, with its homogeneous expansion $f=\sum F_{m}$. Then

$$
\sum_{m=0}^{\infty}\left\|F_{m}\right\|_{\infty} \leq C_{1}\left\|D^{n+q} f\right\|_{1, q} .
$$

In particular, if $f \in A_{q, n+q}^{1}$ then $f$ has a continuous extension to $\partial B$.

We refer to [1] for other possible extensions of this corollary.

4. Multipliers. In this section we study multipliers from certain weighted Bergman spaces into certain spaces of sequences or other weighted Bergman spaces.

As (2.2) shows, the Hilbert space $A_{q}^{2}$ can be identified with a space of sequences from $\mathbb{Z}_{+}^{n}$. This motivates the following definition: For $p \geq 0$, we define $\ell^{p}(n)$ as the space of all sequences $\underline{a}=\left(a_{\alpha}\right), \alpha \in \mathbb{Z}_{+}^{n}$, so that

$$
\|\underline{a}\|_{p} \equiv\left\{\sum\left\{\frac{\alpha !}{(n)_{|\alpha|}}\right\}^{p / 2}\left|a_{\alpha}\right|^{p}\right\}^{1 / p}<\infty .
$$

We also make the following definition: Let $\lambda=\left(\lambda_{m}\right), m \in \mathbb{Z}_{+}$, be a fixed sequence in $\mathbb{C}$, and let $f \in H(B)$ with $a_{\alpha}=a_{\alpha}(f), \alpha \in \mathbb{Z}_{+}^{n}$. We define the multiplication operators $\mathcal{M}_{\lambda}$ and $M_{\lambda}$ by $\mathcal{M}_{\lambda} f=\left(\lambda_{|\alpha|} a_{\alpha}\right)$ and by

$$
\left(M_{\lambda} f\right)(z)=\sum \lambda_{|a|} a_{\alpha} z^{\alpha}
$$


as a function in $H(B)$. We equip $H(B)$ with the usual local uniform topology, and let the domains of definition of $\mathcal{M}_{\lambda}$ and $M_{\lambda}$ be

$$
\mathcal{D}\left(\mathcal{M}_{\lambda}\right)=\left\{f \in H(B): \mathcal{M}_{\lambda} f \in \ell^{\infty}(n)\right\}
$$

and

$$
D\left(M_{\lambda}\right)=\left\{f \in H(B): M_{\lambda} f \in H(B)\right\},
$$

respectively. Under these circumstances, the operators $\mathcal{M}_{\lambda}$ and $M_{\lambda}$ are closed. To show this, suppose $f_{m} \in \mathcal{D}\left(\mathcal{M}_{\lambda}\right), f_{m} \rightarrow f$ in the local uniform topology of $H(B)$, and $\underline{b}_{m}=\mathcal{M}_{\lambda} f_{m} \rightarrow \underline{b}$ in $\ell^{\infty}(n)$. Let $a_{\alpha}^{(m)}=a_{\alpha}\left(f_{m}\right)$, $a_{\alpha}=a_{\alpha}(f), \alpha \in \mathbb{Z}_{+}^{n}$. Clearly, $a_{\alpha}^{(m)} \rightarrow a_{\alpha}$ for every $\alpha \in \mathbb{Z}_{+}^{n}$, and thus also $\lambda_{|\alpha|} a_{\alpha}^{(m)} \rightarrow \lambda_{|\alpha|} a_{\alpha}$ for every $\alpha \in \mathbb{Z}_{+}^{n}$. Since also $b_{\alpha}^{(m)}=\lambda_{|\alpha|} a_{\alpha}^{(m)} \rightarrow b_{\alpha}$ for every $\alpha \in \mathbb{Z}_{+}^{n}$, we find that $\underline{b}=\mathcal{M}_{\lambda} f$ and $f \in \mathcal{D}\left(\mathcal{M}_{\lambda}\right)$, proving that $\mathcal{M}_{\lambda}$ is closed. Similarly, if $f_{m} \in D\left(M_{\lambda}\right)$, and $f_{m} \rightarrow f$ and $g_{m}=M_{\lambda} f_{m} \rightarrow g$ in the local uniform topology of $H(B)$, then $g=M_{\lambda} f$ and $f \in D\left(M_{\lambda}\right)$, which proves that $M_{\lambda}$ is closed.

Let $0<p_{j} \leq \infty$ and $q_{j} \geq 0(j=1,2)$. The sequence $\lambda=\left(\lambda_{m}\right)$ is said to be a multiplier of $A_{q_{1}}^{p_{1}}$ into $\ell^{p_{2}}(n)$ if $\mathcal{M}_{\lambda}\left(A_{q_{1}}^{p_{1}}\right) \subset \ell^{p_{2}}(n)$. It is said to be a multiplier of $A_{q_{1}}^{p_{1}}$ into $A_{q_{2}}^{p_{2}}$ if $M_{\lambda}\left(A_{q_{1}}^{p_{1}}\right) \subset A_{q_{2}}^{p_{2}}$. Since the topologies of $A_{q_{j}}^{p_{j}}$ and $\ell^{p_{j}}(n)(j=1,2)$ are stronger than the topologies of $H(B)$ and $\ell^{\infty}(n)$, respectively, we deduce from the above that $\mathcal{M}_{\lambda}$ or $M_{\lambda}$ are closed if $\lambda$ is a multiplier of $A_{q_{1}}^{p_{1}}$ into $\ell^{p_{2}}(n)$ or into $A_{q_{2}}^{p_{2}}$. In particular, by the closed graph theorem, $\mathcal{M}_{\lambda}$ or $M_{\lambda}$ are bounded operators from $A_{q_{1}}^{p_{1}}$ into $\ell^{p_{2}}(n)$ or into $A_{q_{2}}^{p_{2}}$, if $\lambda$ is a multiplier from $A_{q_{1}}^{p_{1}}$ into $\ell^{p_{2}}(n)$ or into $A_{q_{2}}^{p_{2}}$.

When $n=1$, Duren and Shields [4,5] have studied multipliers from $A_{0}^{p_{1}}(\Delta)$ into $\ell^{p_{2}}(1)$ or into $A_{0}^{p_{2}}(\Delta)$. Here we shall extend the results in $[4$, 5] to $n \geq 1$ and $q_{j} \geq 0(j=1,2)$. To this end, we shall need the following lemma whose proof may be found in Duren [3, p. 101].

LEMMA 4.1. Let $s>t>0$ and let $b_{m} \geq 0$ for $m=1,2, \ldots$ Then

$$
\sum_{m=1}^{N} m^{s} b_{m}=O\left(N^{t}\right) \quad \text { if and only if } \sum_{m=N}^{\infty} b_{m}=O\left(N^{t-s}\right) .
$$

ThEOREM 4.2. Let $q \geq 0,0<p \leq 1$ and $p \leq r<\infty$.

(i) If also $(p, q) \neq(1,0)$, then $\lambda=\left(\lambda_{m}\right)$ is a multiplier from $A_{q}^{p}$ into $\ell^{r}(n)$ if

$$
\sum_{m=1}^{N} m^{r(n+q) / p+d(n-1)}\left|\lambda_{m}\right|^{r}=O\left(N^{r n}\right)
$$

where $d=\max (1, r)$. 
(ii) Conversely, if $\lambda=\left(\lambda_{m}\right)$ is a multiplier from $A_{q}^{p}$ into $\ell^{r}(n)$ then

$$
\sum_{n=1}^{N} m^{r(n+q) / p+r(n-1) / 2}\left|\lambda_{m}\right|^{r}=O\left(N^{r n}\right) .
$$

Proof. Let $f \in A_{q}^{p}$ with $a_{\alpha}=a_{\alpha}(f), \alpha \in \mathbb{Z}_{+}^{n}$, and let $\delta=r[(n+q) / p-n]$. By Theorem 1.2

$$
\int_{0}^{1}(1-\rho)^{\delta-1} M_{1}^{r}(f: \rho) d \rho \leq c_{1}\|f\|_{p, q}^{r}
$$

where $c_{1}=c_{1}(p, q, r)>0$ is a constant. In particular, for any increasing sequence $\left(s_{m}\right)$ with $s_{0}=0$ and $\lim _{m \rightarrow \infty} s_{m}=1$, we have

$$
\begin{aligned}
c_{1}\|f\|_{p, q}^{r} & \geq \sum_{m=0}^{\infty} \mathcal{M}_{1}^{r}\left(f: s_{m}\right) \int_{s_{m}}^{s_{m+1}}(1-\rho)^{\delta-1} d \rho \\
& =\frac{1}{\delta} \sum_{m=0}^{\infty}\left\{\left(1-s_{m}\right)^{\delta}-\left(1-s_{m+1}\right)^{\delta}\right\} \mathcal{M}_{1}^{r}\left(f: s_{m}\right) .
\end{aligned}
$$

Now, by Lemma 4.1, the condition in (i) is equivalent to

$$
\sum_{m=N}^{\infty} m^{d(n-1)}\left|\lambda_{m}\right|^{r}=O\left(N^{-\delta}\right),
$$

and thus, without loss of generality we may assume that $\lambda_{m}>0$ and that

$$
\sum_{m=1}^{\infty} m^{d(n-1)} \lambda_{m}^{r}=1
$$

Moreover, (4.1) also allows us to specify the above sequence $\left(s_{m}\right)$ by letting

$$
\left(1-s_{m}\right)^{\delta}=\sum_{k=m}^{\infty} k^{d(n-1)} \lambda_{k}^{r} \quad(m=0,1, \ldots) .
$$

It follows from Theorem 2.9 that

$$
\begin{aligned}
c_{1}\|f\|_{p, q}^{r} & \geq \frac{1}{\delta} \sum_{m=0}^{\infty} m^{d(n-1)} \lambda_{m}^{r} M_{1}^{r}\left(f: s_{m}\right) \\
& \geq \frac{c_{2}}{\delta} \sum_{m=1}^{\infty} s_{m}^{r m}(m+1)^{r q / 2} \sum_{|\alpha|=m}\left\{\frac{\alpha !}{(n+q)_{|\alpha|}}\right\}^{r / 2}\left|\lambda_{|\alpha|} a_{\alpha}\right|^{r} \\
& =\frac{c_{2}}{\delta} \sum_{m=1}^{\infty} s_{m}^{r m}\left\{\frac{(m+1)^{q}(n)_{m}}{(n+q)_{m}}\right\}^{r / 2} \sum_{|\alpha|=m}\left\{\frac{\alpha !}{(n)_{|\alpha|}}\right\}^{r / 2}\left|\lambda_{|\alpha|} a_{\alpha}\right|^{r}
\end{aligned}
$$


where $c_{2}=c_{2}(q, r)>0$ is a constant. We now observe that $s_{0}=s_{1}=0$, and that by $(4.1)$

$$
\left(1-s_{m}\right)^{\delta}=\sum_{k=m}^{\infty} k^{d(n-1)} \lambda_{k}^{r} \leq c_{3}^{\delta} m^{-\delta}
$$

where $c_{3}=c_{3}(p, q, r)>0$ is a constant. Thus, $s_{m} \geq 1-c_{3} / m$ for $m=$ $2,3, \ldots$, and so $s_{m}^{r m} \geq\left(1-c_{3} / m\right)^{m r} \rightarrow e^{-c_{3} r}>0$. It follows from this and Lemma 2.3 that $\mathcal{M}_{\lambda} f \in \ell^{r}(n)$, and thus (i) follows.

To prove (ii), we use the fact that $\mathcal{M}_{\lambda}$ is a bounded operator from $A_{q}^{p}$ into $\ell^{r}(n)$. Let $h=(n+q) / p+n$, and define $f_{0}(z)=(1-\langle z, \zeta\rangle)^{-h}$, where $\zeta$ is a fixed point in $B$ with $\|\zeta\|=\rho$. It follows that

$$
\left\|\mathcal{M}_{\lambda} f_{0}\right\|_{r} \leq c_{1}\left\|f_{0}\right\|_{p, q}=c_{1}\left\{F\left(p h / 2, p h / 2 ; n+q: \rho^{2}\right)\right\}^{1 / p},
$$

where $c_{1}=\left\|\mathcal{M}_{\lambda}\right\|$ is the quasi-norm of $\mathcal{M}_{\lambda}$ as an operator from $A_{q}^{p}$ into $\ell^{r}(n)$ and where $F(a, b ; c: x)$ is the familiar hypergeometric function

$$
F(a, b ; c: x)=\sum_{m=0}^{\infty} \frac{(a)_{m}(b)_{m}}{m !(c)_{m}} x^{m} .
$$

Thus, by using the Gauss identity

$$
F(a, b ; c: x)=(1-x)^{c-a-b} F(c-a, c-b ; c: x),
$$

we deduce that

$$
\left\|\mathcal{M}_{\lambda} f_{0}\right\|_{r} \leq c_{2}\left(1-\rho^{2}\right)^{-n}
$$

where $c_{2}=c_{2}(\lambda, h, q)>0$ is a constant. It follows that

$$
\sum_{m=1}^{\infty}\left\{\frac{(h)_{m}^{2}\left|\lambda_{m}\right|^{2}}{(n)_{m} m !}\right\}^{r / 2} \rho^{m r} \sum_{|\alpha|=m}\left(\frac{m !}{\alpha !}\right)^{r / 2}\left|\zeta_{0}^{\alpha}\right|^{r}=O\left((1-\rho)^{-r n}\right)
$$

where $\zeta_{0} \in \partial B$. By truncating the series at $m=N$ and putting $\rho=1-1 / N$, we obtain, using Lemma 2.3,

$$
\sum_{m=1}^{N} m^{r h-r(n+1) / 2}\left|\lambda_{m}\right|^{r} \sum_{|\alpha|=m}\left(\frac{m !}{\alpha !}\right)^{r / 2}\left|\zeta_{0}^{\alpha}\right|^{r}=O\left(N^{r n}\right) .
$$

and thus, since $h=(n+q) / p+n$,

$$
\sum_{m=1}^{N} m^{r(n+q) / p+r(n-1) / 2}\left|\lambda_{m}\right|^{r} \sum_{|\alpha|=m}\left(\frac{m !}{\alpha !}\right)^{r / 2}\left|\zeta_{0}^{\alpha}\right|^{r}=O\left(N^{r n}\right) .
$$

The theorem now follows by letting $\zeta_{0}=(1,0, \ldots, 0)$.

Remarks. Some remarks concerning the above theorem are in order:

1. If $n=1,0<p<1$ and $q=0$ or $q=1 / p-1$, then the theorem reduces to a result of Duren and Shields $[4,5]$. Unfortunately, when $n>1$, there 
is a gap between the exponents in the two conditions of the theorem. One possibility of narrowing, or even removing, this gap is to choose the point $\zeta_{0} \in \partial B$ in (4.4) to be different than a unit vector along one of the axes when $r<1$, but we were not able to find such an optimal point.

2. The multipliers from $A_{0}^{1}$ into $\ell^{1}(n)$ are difficult to describe even when $n=1$ (see Duren [3, p. 105]). The reason for the collapse of the sufficient condition (i) of the theorem, when $p=1$ and $q=0$, is the fact that the value $\delta=(n+q) / p-n$ in (4.1) becomes zero in this extreme case. However, by relaxing this exponent, we may obtain the next result which is somewhat weaker.

TheOREM 4.3. Let $1 \leq r<\infty$. Then $\lambda=\left(\lambda_{m}\right)$ is a multiplier from $A_{0}^{1}$ into $\ell^{r}(n)$ if for some $\varepsilon>0$

$$
\sum_{m=1}^{N} m^{r n+d(n-1)+\varepsilon}\left|\lambda_{m}\right|^{r}=O\left(N^{r n}\right)
$$

where $d=\max (1, r)$.

Proof. By Theorem 4.2 (i), $\lambda$ is a multiplier from $A_{\varepsilon / r}^{1}$ into $\ell^{r}(n)$, and since $A_{0}^{1}$ is contained in $A_{\varepsilon / r}^{1}$, it is also a multiplier from $A_{0}^{1}$ into $\ell^{r}(n)$. This concludes the proof.

As in the case $n=1$, it is possible to characterize all the multipliers $\underline{\lambda}=\left(\lambda_{\alpha}\right), \alpha \in \mathbb{Z}_{+}^{n}$, from $A_{0}^{1}$ to $\ell^{1}(n), n \geq 1$, but only by an implicit condition that is not easily verifiable. However, this condition is of interest on its own right, and hence it is perhaps worthwhile presenting it as a theorem (see Theorem 4.7 below). To this end we shall need the following factorization theorem due to Coifman, Rochberg and Weiss [2]:

THEOREM 4.4. There exists a constant $c=c(n)>0$ such that for any $f \in A_{0}^{1}$ there are $g_{m}, h_{m}$ in $A_{0}^{2}$ such that $f=\sum_{m=1}^{\infty} g_{m} h_{m}$ and

$$
\sum_{m=1}^{\infty}\left\|g_{m}\right\|_{2,0}\left\|h_{m}\right\|_{2,0} \leq c\|f\|_{1,0} .
$$

The results described below are classical for $n=1$ (see [3, pp. 47-52]). For simplicity, we drop the index $q$ from $\phi_{\alpha, q}, c_{\alpha}(q), k_{q}, P_{q}$ and $\langle,\rangle_{q}$ when $q=0$. By $\Lambda=\Lambda(B)$ we denote the space of functions in $H(B)$ with bounded mean oscillation (BMO). As is well-known [2], this space may be identified with the Banach space $P\left(L_{0}^{\infty}\right)$ under the norm $\|\cdot\|_{*}$, given by

$$
\|g\|_{*}=\min \left\{\|\psi\|_{\infty, 0}: P \psi=g, \psi \in L_{0}^{\infty}\right\} \quad(g \in \Lambda) .
$$

In particular, $\|P \psi\|_{*} \leq\|\psi\|_{\infty, 0}$ for every $\psi \in L_{0}^{\infty}$, and by Theorem 1.3, $\Lambda \subset A_{0}^{p}$ for every $0<p<\infty$. 
Theorem 4.5. Let $h \in \Lambda$ and $\lambda_{\alpha}=c_{\alpha}^{-1}\left\langle\phi_{\alpha}, h\right\rangle\left(\alpha \in \mathbb{Z}_{+}^{n}\right)$. For $\underline{a}=\left(a_{\alpha}\right)$, $\underline{b}=\left(b_{\alpha}\right), \alpha \in \mathbb{Z}_{+}^{n}$, in $\ell^{2}(n)$, define

$$
Q(\underline{a}, \underline{b})=\sum_{\alpha, \beta} \lambda_{\alpha+\beta} a_{\alpha} b_{\beta} .
$$

Then

$$
|Q(\underline{a}, \underline{b})| \leq\|h\|_{*}\|a\|_{2}\|b\|_{2} .
$$

Proof. Let $f$ and $g$ be the functions in $A_{0}^{2}$ whose Taylor coefficients are $a_{\alpha}(f)=a_{\alpha}, a_{\alpha}(g)=b_{\alpha}, \alpha \in \mathbb{Z}_{+}^{n}$, respectively. Clearly, by $(2.2),\|\underline{a}\|_{2}=$ $\|f\|_{2,0}$ and $\|\underline{b}\|_{2}=\|g\|_{2,0}$. Moreover, $Q(\underline{a}, \underline{b})=\langle f g, h\rangle$. Since $h \in \Lambda$, there exists a sequence $\left(\psi_{m}\right)$ in $L_{0}^{\infty}$ so that $h=P \psi_{m}$ and $\lim _{m \rightarrow \infty}\left\|\psi_{m}\right\|_{\infty, 0}=$ $\|h\|_{*}$. It follows that

$$
\begin{aligned}
|Q(\underline{a}, \underline{b})| & =\left|\left\langle f g, P \psi_{m}\right\rangle\right|=\left|\left\langle f g, \psi_{m}\right\rangle\right| \leq\left\|\psi_{m}\right\|_{\infty, 0}\|f g\|_{1,0} \\
& \leq\left\|\psi_{m}\right\|_{\infty, 0}\|f\|_{2,0}\|g\|_{2,0}=\left\|\psi_{m}\right\|_{\infty, 0}\|\underline{a}\|_{2}\|\underline{b}\|_{2},
\end{aligned}
$$

and letting $m \rightarrow \infty$ concludes the proof.

THEOREM 4.6. Let $\underline{\lambda}=\left(\lambda_{\alpha}\right)$ be a non-negative sequence with $\lambda_{\alpha}=$ $c_{\alpha}^{-1}\left\langle\phi_{\alpha}, h\right\rangle, \alpha \in \mathbb{Z}_{+}^{n}$, for some $h \in \Lambda$. Let $f \in A_{0}^{1}$ with $a_{\alpha}=a_{\alpha}(f), \alpha \in \mathbb{Z}_{+}^{n}$. Then

$$
\sum \lambda_{\alpha}\left|a_{\alpha}\right| \leq c\|h\|_{*}\|f\|_{1,0} .
$$

Proof. By Theorem 4.4, $f=\sum_{m=1}^{\infty} f_{m}$ where $f_{m}=g_{m} h_{m}$ with $g_{m}$, $h_{m}$ in $A_{0}^{2}$ and

$$
\sum_{m=1}^{\infty}\left\|g_{m}\right\|_{2,0}\left\|h_{m}\right\|_{2,0} \leq c\|f\|_{1,0} .
$$

Let $a_{\alpha}^{(m)}=a_{\alpha}\left(f_{m}\right), b_{\alpha}^{(m)}=a_{\alpha}\left(g_{m}\right), d_{\alpha}^{(m)}=a_{\alpha}\left(h_{m}\right)$ be the Taylor coefficients of $f_{m}, g_{m}, h_{m}, m=1,2, \ldots$, respectively. Then

$$
a_{\alpha}^{(m)}=\sum_{\beta \leq \alpha} b_{\beta} d_{\alpha-\beta},
$$

and by Theorem 4.5 ,

$$
\begin{aligned}
\sum_{\alpha} \lambda_{\alpha}\left|a_{\alpha}\right| & \leq \sum_{m=1}^{\infty} \sum_{\alpha} \lambda_{\alpha}\left|a_{\alpha}^{(m)}\right| \leq \sum_{m=1}^{\infty} \sum_{\alpha} \lambda_{\alpha} \sum_{\beta \leq \alpha}\left|b_{\beta}^{(m)}\right|\left|d_{\alpha-\beta}^{(m)}\right| \\
& \leq \sum_{m=1}^{\infty} \sum_{\alpha, \beta} \lambda_{\alpha+\beta}\left|b_{\alpha}^{(m)}\right|\left|d_{\beta}^{(m)}\right| \leq \sum_{m=1}^{\infty}\|h\|_{*}\left\|\underline{b}^{(m)}\right\|_{2}\left\|\underline{d}^{(m)}\right\|_{2} \\
& =\|h\|_{*} \sum_{m=1}^{\infty}\left\|g_{m}\right\|_{2,0}\left\|h_{m}\right\|_{2,0} \leq c\|h\|_{*}\|f\|_{1,0} .
\end{aligned}
$$

The proof is now complete. 
As before, for a sequence $\underline{\lambda}=\left(\lambda_{\alpha}\right)$ and a function $f$ in $H(B)$ with $a_{\alpha}=a_{\alpha}(f), \alpha \in \mathbb{Z}_{+}^{n}$, we define $\mathcal{M}_{\underline{\lambda}} f=\left(\lambda_{\alpha} a_{\alpha}\right)$. The sequence $\underline{\lambda}$ is a multiplier of $A_{0}^{1}$ into $\ell^{1}(n)$ if $\mathcal{M}_{\underline{\lambda}}$ maps $A_{0}^{1}$ into $\ell^{1}(n)$. In this case, again by the closed graph theorem, $\mathcal{M}_{\underline{\lambda}}^{\underline{\lambda}}$ is a bounded operator from $A_{0}^{1}$ into $\ell^{1}(n)$ with norm $\left\|\mathcal{M}_{\underline{\lambda}}\right\|$.

THEOREM 4.7. The sequence $\underline{\lambda}=\left(\lambda_{\alpha}\right)$ is a multiplier of $A_{0}^{1}$ into $\ell^{1}(n)$ if and only if there is a function $h \in \Lambda$ such that $\left|\lambda_{\alpha}\right|=\left\langle\phi_{\alpha}, h\right\rangle\left(\alpha \in \mathbb{Z}_{+}^{n}\right)$.

Proof. The sufficiency of the condition follows from Theorem 4.6. Indeed, for $f \in A_{0}^{1}$ with $a_{\alpha}=a_{\alpha}(f), \alpha \in \mathbb{Z}_{+}^{n}$, the above theorem gives

$$
\left\|\mathcal{M}_{\underline{\lambda}} f\right\|_{1}=\sum c_{\alpha}^{-1}\left|\lambda_{\alpha}\right|\left|a_{\alpha}\right| \leq c\|h\|_{*}\|f\|_{1,0} .
$$

Conversely, suppose $\underline{\lambda}=\left(\lambda_{\alpha}\right)$ is a multiplier from $A_{0}^{1}$ into $\ell^{1}(n)$. Thus $\left\|\mathcal{M}_{\underline{\lambda}} f\right\|_{1} \leq\left\|\mathcal{M}_{\underline{\lambda}}\right\|\|f\|_{1,0}$ or

$$
\sum c_{\alpha}^{-1}\left|\lambda_{\alpha}\right|\left|a_{\alpha}\right| \leq\left\|\mathcal{M}_{\underline{\lambda}}\right\|\|f\|_{1,0}
$$

for every $f \in A_{0}^{1}$ with $a_{\alpha}=a_{\alpha}(f), \alpha \in \mathbb{Z}_{+}^{n}$. In particular, $T$ defined by

$$
T f=\sum c_{\alpha}^{-1}\left|\lambda_{\alpha}\right| a_{\alpha}
$$

is a bounded linear functional on $A_{0}^{1}$. By the Hahn-Banach theorem, $T$ can be extended to a bounded linear functional $\widetilde{T}$ on $L_{0}^{1}$ with $\|\widetilde{T}\|=\|T\| \leq$ $\left\|\mathcal{M}_{\underline{\lambda}}\right\|$. It follows from the Riesz representation theorem that there exists a unique $\psi \in L_{0}^{\infty}$ with $\widetilde{T} f=\langle f, \psi\rangle$ for every $f \in L_{0}^{1}$ and $\|\widetilde{T}\|=\|\psi\|_{\infty, 0}$. In particular, $T f=\langle f, \psi\rangle=\langle f, P \psi\rangle$ for every $f \in A_{0}^{2}$. Letting $h=P \psi$, we find that $h \in \Lambda$ with $\|h\|_{*} \leq\|\psi\|_{\infty, 0} \leq\left\|\mathcal{M}_{\lambda}\right\|$, and $T f=\langle f, h\rangle$ for every $f \in A_{0}^{2}$. Choosing $f \in A_{0}^{2}$ as $f=c_{\alpha}^{-1} \phi_{\alpha}$ gives $\left|\lambda_{\alpha}\right|=\left\langle\phi_{\alpha}, h\right\rangle$ for every $\alpha \in \mathbb{Z}_{+}^{n}$, and the theorem is proved.

We remark that in Theorems 4.5, 4.6 and 4.7, the function $h \in \Lambda$ with $\|h\|_{*}$ can be replaced by $\psi \in L_{0}^{\infty}$ with $\|\psi\|_{\infty, 0}$.

We now describe multipliers from $A_{q_{1}}^{p_{1}}$ into $A_{q_{2}}^{p_{2}}$. For this purpose, we shall prepare some auxiliary lemmas.

Lemma 4.8. Let $\mu \in L^{1}(0,1)$ be continuous and positive on $(0,1)$. Then there is a constant $c=c(\mu)>0$ such that

$$
\int_{0}^{1} g(\rho) \mu(\rho) d \rho \leq c \int_{1 / 2}^{1} g(\rho) \mu(\rho) d \rho
$$

for every non-negative increasing function $g$ on $(0,1)$. 
Proof. We have

$$
\begin{aligned}
\int_{0}^{1 / 2} g(\rho) \mu(\rho) d \rho & \leq g(1 / 2) \int_{0}^{1 / 2} \mu(\rho) d \rho=A \cdot g(1 / 2) \leq 4 A \int_{1 / 2}^{3 / 4} g(\rho) d \rho \\
& \leq 4 A A_{1} \int_{1 / 2}^{3 / 4} g(\rho) \mu(\rho) d \rho \leq 4 A A_{1} \int_{1 / 2}^{1} g(\rho) \mu(\rho) d \rho
\end{aligned}
$$

where $A=\int_{1 / 2}^{1} \mu(\rho) d \rho$ and $A_{1}^{-1}=\min \{\mu(\rho): 1 / 2 \leq \rho \leq 3 / 4\}$. Thus

$$
\int_{0}^{1} g(\rho) \mu(\rho) d \rho \leq\left(1+4 A A_{1}\right) \int_{1 / 2}^{1} g(\rho) \mu(\rho) d \rho,
$$

concluding the proof.

Corollary 4.9. Let $0<p<\infty, q>0, s>0$ and $t \geq 0$. Then there is a constant $c=c(q, s)>0$ such that

$$
\int_{0}^{1}(1-\rho)^{q-1} M_{p}^{t}(f: \rho) d \rho \leq c \int_{0}^{1}(1-\rho)^{q-1} \rho^{s} M_{p}^{t}(f: \rho) d \rho
$$

for every $f \in H(B)$.

LEMMA 4.10. Let $p \geq 1$ and $q>0$. Then there is a constant $c=c(q)>0$ such that

$$
\begin{gathered}
\|f\|_{p, 0} \leq \int_{0}^{1} M_{p}(D f: r) d r, \quad \text { and } \\
\int_{0}^{1}(1-r)^{q-1} M_{p}(f: r) d r \leq c \int_{0}^{1}(1-r)^{q} M_{p}(D f: r) d r
\end{gathered}
$$

for every $f \in H(B)$.

Proof. Let $f \in H(B)$ and $0<r<1$. One then verifies that $(D f)(r z)=$ $(d / d r)(r f(r z)), z \in \bar{B}$, and thus

$$
r f(r z)=\int_{0}^{r}(D f)(\rho z) d \rho \quad(z \in \bar{B}) .
$$

It follows from Minkowski's inequality for integrals that

$$
r M_{p}(f: r)=\left(\int_{\partial B}\left|\int_{0}^{r}(D f)(\rho z) d \rho\right|^{p} d v_{0}(z)\right)^{1 / p}
$$




$$
\leq \int_{0}^{r}\left(\int_{\partial B}|(D f)(\rho z)|^{p} d v_{0}(z)\right)^{1 / p} d \rho=\int_{0}^{r} M_{p}(D f: \rho) d \rho .
$$

This gives the first inequality of the lemma, at once. Moreover, by Corollary 4.9 , there is a constant $c_{1}(q)>0$ so that

$$
\begin{aligned}
\int_{0}^{1}(1-r)^{q-1} M_{p}(f: r) d r & \leq c_{1}(q) \int_{0}^{1}(1-r)^{q-1} r M_{p}(f: r) d r \\
& \leq c_{1}(q) \int_{0}^{1}(1-r)^{q-1}\left(\int_{0}^{r} M_{p}(D f: \rho) d \rho\right) d r \\
& =c_{1}(q) \int_{0}^{1} M_{p}(D f: \rho)\left(\int_{0}^{\rho}(1-r)^{q-1} d r\right) d \rho \\
& =q^{-1} c_{1}(q) \int_{0}^{1}(1-\rho)^{q} M_{p}(D f: \rho) d \rho,
\end{aligned}
$$

and the proof is complete.

For a sequence $\lambda=\left(\lambda_{m}\right)$ and $z, \zeta \in B$, we let $H_{\lambda}(z, \zeta)=h_{\lambda}(\langle z, \zeta\rangle)$ where

$$
h_{\lambda}(t)=\sum_{m=0}^{\infty} \frac{(n)_{m}}{m !} \lambda_{m} t^{m} \quad(t \in \Delta) .
$$

The proof of the next lemma is immediate:

Lemma 4.11. Let $\lambda=\left(\lambda_{m}\right)$ be a sequence in $\mathbb{C}, s \in \mathbb{R}$ and $0<\rho<1$. Then

$$
\left(D^{s} M_{\lambda} f\right)(\rho z)=\int_{\partial B} f(\rho \zeta) D_{z}^{s} H_{\lambda}(z, \zeta) d v_{0}(\zeta) \quad(z \in \bar{B})
$$

for every $f \in H(B)$.

We are now in a position to state and prove the following result:

Theorem 4.12. Suppose $0<p_{1}<1 \leq p_{2}<\infty$ and $q_{1}, q_{2} \geq 0$, and let $\ell$ be the integer part of $n\left(1-1 / p_{1}\right)+q_{1} / p_{1}+1$. Then $\lambda=\left(\lambda_{m}\right)$ is a multiplier from $A_{q_{1}}^{p_{1}}$ into $A_{q_{2}}^{p_{2}}$ if and only if

$$
M_{p_{2}}\left(D^{\ell+1} H_{\lambda}(\cdot, \zeta): r\right)=O\left((1-r)^{\left(n+q_{1}\right) p_{1}-n-\ell-q_{2} / p_{2}}\right)
$$

for every $\zeta \in \partial B$.

Proof. For the necessity we consider the sequence $\delta=\left((m+1)^{\ell+1}\right)$ and a fixed point $\zeta \in B$, and define $f_{\zeta}=H_{\delta}(\cdot, \zeta)$. Thus, $f_{\zeta}$ is in $H(B)$ with $M_{\lambda} f_{\zeta}=D^{\ell+1} H_{\lambda}(\cdot, \zeta)$, and so,

$$
\left\|M_{\lambda} f_{\zeta}\right\|_{p_{2}, q_{2}} \leq\left\|M_{\lambda}\right\|\left\|f_{\zeta}\right\|_{p_{1}, q_{1}} .
$$


On the other hand,

$$
f_{\zeta}(z)=\sum_{m=0}^{\infty} \frac{(n)_{m}}{m !}(m+1)^{\ell+1}\langle z, \zeta\rangle^{m}
$$

and thus (see Beatrous and Burbea [1])

$$
f_{\zeta}(z)=(1-\langle z, \zeta\rangle)^{-(n+\ell+1)} F(\langle z, \zeta\rangle)
$$

where $F \in H(\Delta)$ and of Lipschitz class of order $n+\ell+1$. It follows, as in (3.1) and (4.2)-(4.3), that for $0<r<1$ and $\zeta \in \partial B$

$$
\begin{aligned}
\left\|f_{r \zeta}\right\|_{p_{1}, q_{1}} & \leq c_{1}\left\|k_{\ell+1}(\cdot, r \zeta)\right\|_{p_{1}, q_{1}} \\
& =c_{1}\left\{F\left((n+\ell+1) p_{1} / 2,(n+\ell+1) p_{1} / 2 ; n+q_{1}: r^{2}\right)\right\}^{1 / p_{1}} \\
& \leq c_{2}\left(1-r^{2}\right)^{\left(n+q_{1}\right) / p_{1}-n-\ell-1}
\end{aligned}
$$

where $c_{1}=c_{1}(\ell)>0$ and $c_{2}=c_{2}\left(\ell, p_{1}, q_{1}\right)>0$ are constants. Thus

$$
\left\|D^{\ell+1} H_{\lambda}(\cdot, r \zeta)\right\|_{p_{2}, q_{2}} \leq c_{2}\left\|M_{\lambda}\right\|\left(1-r^{2}\right)^{\left(n+q_{1}\right) / p_{1}-n-\ell-1} \quad(\zeta \in \partial B),
$$

and the result follows when $q_{2}=0$. For $q_{2}>0$, on the other hand, using Corollary 4.9 we have for $\zeta \in \partial B$

$$
\begin{aligned}
M_{p_{2}}\left(D^{\ell+1} H_{\lambda}(\cdot, \zeta)\right. & \left.: r^{2}\right)\left(\int_{r}^{1}(1-\rho)^{q_{2}-1} d \rho\right)^{1 / p_{2}} \\
& \leq\left(\int_{r}^{1}(1-\rho)^{q_{2}-1} M_{q_{2}}^{p_{2}}\left(D^{\ell+1} H_{\lambda}(\cdot, \zeta): r \rho\right) d \rho\right)^{1 / p_{2}} \\
& \leq\left(\int_{0}^{1}(1-\rho)^{q_{2}-1} M_{q_{2}}^{p_{2}}\left(D^{\ell+1} H_{\lambda}(\cdot, r \zeta): \rho\right) d \rho\right)^{1 / p_{2}} \\
& \leq c_{3}\left\|D^{\ell+1} H_{\lambda}(\cdot, r \zeta)\right\|_{p_{2}, q_{2}}
\end{aligned}
$$

where $c_{3}=c_{3}\left(q_{2}, p_{2}\right)>0$ is a constant. It follows that

$$
M_{p_{2}}\left(D^{\ell+1} H_{\lambda}(\cdot, \zeta): r^{2}\right)=O\left(\left(1-r^{2}\right)^{\left(n+q_{1}\right) / p_{1}-n-\ell-1-q_{2} / p_{2}}\right),
$$

which is equivalent to the desired result when $q_{2}>0$.

For the sufficiency, we let $f \in A_{q_{1}}^{p_{1}}$ and $0<\rho<1$, and use Lemma 4.11 to obtain

$$
\left(D^{\ell+1} M_{\lambda} f\right)(\rho z)=\int_{\partial B} f(\rho \zeta) D^{\ell+1} H_{\lambda}(z, \zeta) d v_{0}(\zeta) \quad(z \in \bar{B}) .
$$

It follows from Minkowski's inequality for integrals that

$$
\begin{aligned}
M_{p_{2}} & \left(D^{\ell+1} M_{\lambda} f: r \rho\right) \\
& \leq \int_{\partial B}\left(\int_{\partial B}|f(\rho \zeta)|^{p_{2}}\left|D^{\ell+1} H_{\lambda}(r z, \zeta)\right|^{p_{2}} d v_{0}(z)\right)^{1 / p_{2}} d v_{0}(\zeta)
\end{aligned}
$$




$$
=\int_{\partial B}|f(\rho \zeta)| M_{p_{2}}\left(D^{\ell+1} H(\cdot, \zeta): r\right) d v_{0}(\zeta),
$$

and thus, by assumption, for a constant $c_{1}>0$,

$$
M_{p_{2}}\left(D^{\ell+1} M_{\lambda} f: r \rho\right) \leq c_{1} M_{1}(f: \rho)(1-r)^{\left(n+q_{1}\right) / p_{1}-n-\ell-1-q_{2} / p_{2}} .
$$

We first assume that $q_{2}=0$. In this case, taking $\rho=r$, and using Corollary 4.9 and Theorem 1.2, we have

$$
\begin{aligned}
& \int_{0}^{1}(1-r)^{\ell} M_{p_{2}}\left(D^{\ell+1} M_{\lambda} f: r\right) d r=2 \int_{0}^{1}\left(1-r^{2}\right)^{\ell} r M_{p_{2}}\left(D^{\ell+1} M_{\lambda} f: r^{2}\right) d r \\
& \quad \leq 2^{\ell+1} c_{1} \int_{0}^{1}(1-r)^{\left(n+q_{1}\right) / p_{1}-n-1} r M_{1}(f: r) d r \\
& \quad \leq 2^{\ell+1} c_{1} c_{2} \int_{0}^{1}(1-r)^{\left(n+q_{1}\right) / p_{1}-n-1} M_{1}(f: r) d r \leq 2^{\ell+1} c_{1} c_{3}\|f\|_{p_{1}, q_{1}}
\end{aligned}
$$

where $c_{j}=c_{j}\left(p_{1}, q_{1}\right)>0$ is a constant $(j=2,3)$. By $\ell$ successive applications of Lemma 4.10, we obtain therefore

$$
\left\|M_{\lambda} f\right\|_{p_{2}, 0} \leq \int_{0}^{1} M_{p_{2}}\left(D M_{\lambda} f: r\right) d r \leq c_{1} c_{4}\|f\|_{p_{1}, q_{1}}
$$

where $c_{4}=c_{4}\left(\ell, p_{1}, q_{1}\right)>0$ is a constant. Thus $M_{\lambda} f \in A_{0}^{p_{2}}$, and the result follows for $q_{2}=0$. When, on the other hand, $q_{2}>0$, we have, by using Corollary 4.9 and Theorem 1.2,

$$
\begin{aligned}
& \left\|D^{\ell+1} M_{\lambda} f\right\|_{p_{2}, q_{2}+p_{2}(\ell+1)}^{p_{2}}=\frac{2 \Gamma\left(n+q_{2}+p_{2}(\ell+1)\right)}{\Gamma(n) \Gamma\left(q_{2}+p_{2}(\ell+1)\right)} \\
& \quad \times \int_{0}^{1}\left(1-r^{2}\right)^{q_{2}+p_{2}(\ell+1)-1} r^{2 n-1} M_{p_{2}}^{p_{2}}\left(D^{\ell+1} M_{\lambda} f: r\right) d r \\
& \leq c_{5} \int_{0}^{1}(1-r)^{q_{2}+p_{2}(\ell+1)-1} M_{p_{2}}^{p_{2}}\left(D^{\ell+1} M_{\lambda} f: r^{2}\right) d r \\
& \leq c_{1} c_{5} \int_{0}^{1}(1-r)^{p_{2}\left[\left(n+q_{1}\right) / p_{1}-n\right]-1} M_{1}^{p_{2}}(f: r) d r \\
& \leq c_{1} c_{6}\|f\|_{p_{1}, q_{1}}^{p_{2}}
\end{aligned}
$$

where $c_{j}=c_{j}\left(\ell, p_{1}, p_{2}, q_{1}, q_{2}\right)>0(j=5,6)$ is a constant. It follows that $M_{\lambda} f \in A_{q_{2}+p_{2}(\ell+1), \ell+1}^{p_{2}}$, and thus by Theorem 1.3, also $M_{\lambda} f \in A_{q_{2}}^{p_{2}}$. This concludes the proof. 


\section{References}

[1] F. Beatrous and J. Burbea, Holomorphic Sobolev spaces on the ball, Dissertationes Math. 276 (1989).

[2] R. R. Coifman, R. Rochberg and G. Weiss, Factorization theorems for Hardy spaces in several variables, Ann. of Math. 103 (1976), 611-635.

[3] P. L. Duren, Theory of $H^{p}$ Spaces, Academic Press, New York 1970.

[4] P. L. Duren and A. L. Shields, Properties of $H^{p}(0<p<1)$ and its containing Banach space, Trans. Amer. Math. Soc. 141 (1969), 255-261.

[5] -, 一, Coefficient multipliers of $H^{p}$ and $B^{p}$ spaces, Pacific J. Math. 32 (1970), 69-78.

[6] T. M. Flett, On the rate of growth of mean values of holomorphic and harmonic functions, Proc. London Math. Soc. 20 (1970), 749-768.

[7] F. Forelli and W. Rudin, Projections on the spaces of holomorphic functions in balls, Indiana Univ. Math. J. 24 (1974), 593-602.

[8] G. H. Hardy and J. E. Littlewood, Theorems concerning mean values of analytic or harmonic functions, Quart. J. Math. Oxford Ser. 12 (1942), 221-256.

[9] A. Korányi and S. Vagi, Singular integrals in homogeneous spaces and some problems of classical analysis, Ann. Scuola Norm. Sup. Pisa 25 (1971), 575-648.

[10] E. M. Stein and G. Weiss, On the interpolation of analytic families of operators acting on $H^{p}$ spaces, Tôhoku Math. J. (2) 9 (1957), 318-339.

DEPARTMENT OF MATHEMATICS

UNIVERSITY OF PITTSBURGH PITTSBURGH, PENNSYLVANIA 15260

U.S.A.
KOREA INSTITUTE OF TECHNOLOGY 400 GUSONG-DONG, CHUNG-KU TAEJON, KOREA 300-31 